\title{
UNIFORM LOCAL WELL-POSEDNESS AND REGULARITY CRITERION FOR THE DENSITY-DEPENDENT INCOMPRESSIBLE FLOW OF LIQUID CRYSTALS*
}

\author{
JISHAN FAN ${ }^{\dagger}$ AND FUCAI LI ${ }^{\ddagger}$
}

\begin{abstract}
In this paper we first prove the uniform local well-posedness for the density-dependent incompressible flow of liquid crystals in the whole space $\mathbb{R}^{3}$. Next, we provide a regularity criterion for the strong solution when the initial density may contain vacuum.
\end{abstract}

Key words. Density-dependent incompressible flow, liquid crystals, vacuum, uniform local well-posedness, regularity criterion.

AMS subject classifications. 35Q30, 76D03, 82D30.

\section{Introduction}

In this paper, we consider the following density-dependent liquid crystals system $([1,6,15,19,20])$ :

$$
\begin{aligned}
& \partial_{t} \rho+\operatorname{div}(\rho u)=0, \\
& \partial_{t}(\rho u)+\operatorname{div}(\rho u \otimes u)+\nabla \pi-\mu \Delta u=-\operatorname{div}(\nabla d \odot \nabla d), \\
& \partial_{t} d+u \cdot \nabla d-\Delta d=d|\nabla d|^{2}, \quad|d|=1, \\
& \operatorname{div} u=0,
\end{aligned}
$$

with the initial data

$$
(\rho, u, d)(x, 0)=\left(\rho_{0}, u_{0}, d_{0}\right)(x), \quad\left|d_{0}\right|=1, \quad x \in \mathbb{R}^{3} .
$$

Here $\rho$ denotes the density, $u \in \mathbb{R}^{3}$ the velocity, and $d \in \mathbb{S}^{2}$ (the unit spherical surface in $\mathbb{R}^{3}$ ) the macroscopic molecular orientations, respectively. The constant $\mu>0$ is the viscosity coefficient. The symbol $\nabla d \odot \nabla d$ denotes a matrix whose components are defined as

$$
(\nabla d \odot \nabla d)_{i, j}:=\sum_{k=1}^{3} \partial_{i} d_{k} \partial_{j} d_{k}, \quad 1 \leq i, j \leq 3 .
$$

The term

$$
\operatorname{div}(\nabla d \odot \nabla d)=\sum_{k=1}^{3} \Delta d_{k} \nabla d_{k}+\frac{1}{2} \sum_{k=1}^{3} \nabla\left|\nabla d_{k}\right|^{2}
$$

in (1.2) is a stress tensor which represents the anisotropic feature of the system.

When $d$ is a constant vector in $\mathbb{S}^{2}$, the system (1.1)-(1.4) is reduced to the wellknown density-dependent Navier-Stokes equations and there are many results on this system; see [2-5] among others. Liu.

*Received: May 3, 2013; accepted (in revised form): August 27, 2013. Communicated by Chun

${ }^{\dagger}$ Department of Applied Mathematics, Nanjing Forestry University, Nanjing 210037, P.R. China (fanjishan@njfu.edu.cn).

${ }^{\ddagger}$ Corresponding author. Department of Mathematics, Nanjing University, Nanjing 210093, P.R. China (fli@nju.edu.cn). 
When $\rho \equiv 1$, the system (1.1)-(1.4) becomes the incompressible liquid crystals system and many studies are available [16,18-22]. Recently, Li and Wang [17] studied the system (1.1)-(1.4) in a bounded smooth domain of $\mathbb{R}^{3}$ where the existence and uniqueness of local (global) strong solution were obtained with large (small) initial data when the initial density is away from vacuum. Fan, Gao, and Guo [7] considered the regularity criterion for the system (1.1)-(1.4) with an additional term $d \times \Delta d$ in (1.3) in the whole space $\mathbb{R}^{3}$ when the initial density has a positive bound from below.

In this paper we first establish the uniform local-in-time well-posedness of the problem (1.1)-(1.5), i.e., we obtain estimates which do not depend on $\mu>0$. Our result reads as follows.

Theorem 1.1. Let $0<\mu \leq 1$ and $s>5 / 2$ be two real numbers. Suppose that there exist two positive constants $m$ and $M_{1}$ such that the initial data satisfy

$$
\left\{\begin{array}{l}
m \leq \rho_{0} \leq M_{1}, \quad \nabla \rho_{0} \in H^{s-1}\left(\mathbb{R}^{3}\right), \\
\left(u_{0}, \nabla d_{0}\right) \in H^{s}\left(\mathbb{R}^{3}\right), \quad\left|d_{0}\right|=1, \quad \operatorname{div} u_{0}=0 .
\end{array}\right.
$$

Then there exists a positive time $T^{*}>0$ such that the problem (1.1)-(1.5) has a unique solution $(\rho, u, d)$ satisfying

$$
0<m \leq \rho \leq M_{1}, \quad\|\nabla \rho\|_{L^{\infty}\left(0, T^{*} ; H^{s-1}\left(\mathbb{R}^{3}\right)\right)} \leq C, \quad\|(u, \nabla d)\|_{L^{\infty}\left(0, T^{*} ; H^{s}\left(\mathbb{R}^{3}\right)\right)} \leq C .
$$

Here and in Section 3 below the positive constants $T^{*}$ and $C$ are independent of $\mu$.

REMARK 1.2. By our estimates (1.8), the limit $\mu \rightarrow 0$ can be studied directly. Hence we also obtain the local well-posedness of the problem (1.1)-(1.5) with $\mu=0$. We omit it here for conciseness, and the reader can refer [11] for the corresponding results on the density-dependent incompressible Navier-Stokes equations.

Next, we study the regularity criterion for the system (1.1)-(1.4) in the whole space $\mathbb{R}^{3}$ when the initial density may vanish on an open subset of $\mathbb{R}^{3}$. We assume that the initial data satisfy

$$
\left\{\begin{array}{l}
0 \leq \rho_{0} \leq M_{2}<\infty, \quad \nabla \rho_{0} \in L^{2}\left(\mathbb{R}^{3}\right) \cap L^{q}\left(\mathbb{R}^{3}\right), \quad 3<q \leq 6 \\
u_{0} \in H^{2}\left(\mathbb{R}^{3}\right), \quad \operatorname{div} u_{0}=0, \quad \nabla d_{0} \in H^{2}\left(\mathbb{R}^{3}\right), \quad\left|d_{0}\right|=1
\end{array}\right.
$$

and the compatibility condition

$$
\Delta u_{0}-\operatorname{div}\left(\nabla d_{0} \odot \nabla d_{0}\right)-\nabla \pi_{0}=\rho_{0}^{1 / 2} g \text { for some } g \in L^{2}\left(\mathbb{R}^{3}\right) .
$$

Under the conditions (1.9) and (1.10), it is easy to prove that there is a constant $T_{0}>0$ such that the problem (1.1)-(1.5) has a unique strong solution $(\rho, u, \pi, d)$ in $\left(0, T_{0}\right]$; for example, see Kim [14] on the density-dependent incompressible Navier-Stokes system. However, the global-in-time regularity of the problem (1.1)-(1.5) is still open. Here we provide a regularity criterion for the strong solutions when the initial density may contain vacuum. Our result can be stated as follows.

TheOREm 1.3. Suppose that the conditions (1.9) and (1.10) hold. Let $(\rho, u, d)$ be a strong solution to the problem (1.1)-(1.5). If the following conditions hold:

$$
\begin{aligned}
& u \in L^{r}\left(0, T ; L^{s}\left(\mathbb{R}^{3}\right)\right) \text { with } \frac{2}{r}+\frac{3}{s}=1, \quad 3<s \leq \infty, \\
& \nabla d \in L^{2}\left(0, T ; \dot{B}_{\infty, \infty}^{0}\left(\mathbb{R}^{3}\right)\right),
\end{aligned}
$$


then the solution $(\rho, u, \pi, d)$ can be extended beyond $T$, where the symbol $\dot{B}_{\infty, \infty}^{0}$ denotes the homogeneous Besov space.

REMARK 1.4. If $(\rho, u, d)$ is a solution to the problem (1.1)-(1.5), then so does $\left(\rho_{\lambda}, u_{\lambda}, d_{\lambda}\right):=(\rho, \lambda u, d)\left(\lambda x, \lambda^{2} t\right)$. In this sense, our conditions (1.11) and (1.12) are optimal.

REMARK 1.5. It should be pointed out that a variant of the blow-up criterion (1.11)-(1.12) for compressible liquid crystals flow was obtained recently by Huang, Wang, and Wen [10].

We give some comments on the proofs of our results. Because the local-in-time well-posedness has been proved in [17], to complete the proof of Theorem 1.1 we only need to prove the a priori estimates (1.8). We shall employ an elaborate nonlinear energy method to obtain these desired bounds. More precisely, we first derive an energy estimate based on the $L^{2}$ energy of the system. Next, we use Sobolev imbedding, bilinear commutator estimates, and the Gagliardo-Nirenberg inequality to obtain higher order estimates on the density $\rho$, the velocity $u$, and the unit vector field $d$, which satisfies a differential inequality. Then our results come from an application of the well-known Osgood lemma. To prove Theorem 1.3, by the local existence and uniqueness result, and bootstrapping arguments, we only need to establish sufficient regularity estimates on the solutions which can be obtained by Sobolev imbedding, the Gagliardo-Nirenberg inequality, and the conditions (1.11)-(1.12).

This paper is organized as follows. In Section 2, we give some notations and recall some basic inequalities. The proofs of Theorem 1.1 and Theorem 1.3 are presented in sections 3 and 4, respectively.

\section{Preliminaries}

In this section, we give some notations and recall some basic inequalities which will be used frequently. The symbol $\|\cdot\|_{L^{p}}$ standards for the norm in the Lebesgue space $L^{p}\left(\mathbb{R}^{3}\right)$ and $\|\cdot\|_{W^{k, p}}$ for the Sobolev space $W^{k, p}\left(\mathbb{R}^{3}\right)$ (here $k$ is an integer). When $p=2$, we use $\|\cdot\|_{H^{k}}$ for the Sobolev space $W^{k, 2}\left(\mathbb{R}^{3}\right)$. We use $C$ and $M_{i}$ to denote the positive constants which are independent of $\mu$ and may change from line to line. We also omit the spatial domain $\mathbb{R}^{3}$ in the integrals below for simplicity.

First, we introduce the following Osgood lemma [8].

Lemma 2.1 (Osgood lemma). Let $y$ be a measurable, positive function, $f$ a positive, locally integrable function and $g$ a continuous increasing function. Assume that, for a positive real number a, the function y satisfies

$$
y(t) \leq a+\int_{t_{0}}^{t} f(s) g(y(s)) \mathrm{ds}
$$

If $a$ is zero and $g(s)$ satisfies

$$
\int_{0}^{1} \frac{\mathrm{d} r}{g(r)}=+\infty
$$

then the function $y$ is identically zero. If a is different from zero, then we have

$$
-G(y(t))+G(a) \leq \int_{t_{0}}^{t} f(s) \mathrm{d} s \text { with } \quad G(s):=\int_{s}^{1} \frac{\mathrm{d} r}{g(r)} .
$$


Next, we recall the well-known Gagliardo-Nirenberg inequality $[9,25]$.

LEMMA 2.2 (Gagliardo-Nirenberg inequality). Let $v \in W^{k, r}\left(\mathbb{R}^{3}\right) \cap L^{q}\left(\mathbb{R}^{3}\right), 1 \leq$ $q, r \leq \infty$. Then the following inequalities hold:

$$
\left\|D^{i} v\right\|_{L^{p}} \leq M_{0}\left\|D^{k} v\right\|_{L^{r}}^{\alpha}\|v\|_{L^{q}}^{1-\alpha}, \quad \forall 0 \leq i<k,
$$

where

$$
\frac{1}{p}=\frac{i}{3}+\alpha\left(\frac{1}{r}-\frac{k}{3}\right)+(1-\alpha) \frac{1}{q},
$$

for all $\alpha$ in the interval

$$
\frac{i}{k} \leq \alpha \leq 1
$$

The constant $M_{0}$ depends only on $d, m, j, q, r$, and $\alpha$, with the following exceptional case:

1. If $i=0, r k<3$, and $q=\infty$ then we make the additional assumption that either $v$ tends to zero at infinity or $v \in L^{\tilde{q}}\left(\mathbb{R}^{N}\right)$ for some finite $\tilde{q}>0$.

2. If $1<r<\infty$, and $k-i-N / r$ is a non negative integer then (2.1) holds only for $\alpha$ satisfying $i / k \leq \alpha<1$.

By the Gagliardo-Nirenberg inequality (2.1) and Sobolev imbedding, we have the following inequalities which will be used frequently:

$$
\begin{aligned}
& \|\nabla v\|_{L^{2 p}}^{2} \leq C\|v\|_{L^{\infty}}\|\Delta v\|_{L^{p}} \text { for } 1<p<\infty \\
& \|v\|_{L^{\frac{2 s}{s-2}}} \leq C\|v\|_{L^{2}}^{1-3 / s}\|\nabla v\|_{L^{2}}^{3 / s} \text { for } 3<s \leq \infty \\
& \|\nabla v\|_{L^{3}}^{2} \leq C\|\nabla v\|_{L^{2}}\|\Delta v\|_{L^{2}} \\
& \|v\|_{L^{\infty}}^{2} \leq C\|v\|_{L^{6}}\|\Delta v\|_{L^{2}} \leq C\|\nabla v\|_{L^{2}}\|\Delta v\|_{L^{2}}, \\
& \|\nabla d\|_{L^{\infty}}^{2} \leq C\|\nabla d\|_{L^{6}}\|\nabla \Delta d\|_{L^{2}} .
\end{aligned}
$$

Third, we define the operator $\Lambda:=(-\Delta)^{1 / 2}$ via the Fourier transform

$$
\widehat{\Lambda f}(\xi)=|\xi| \hat{f}(\xi) .
$$

Generally, we define $\Lambda^{s} f$ for $s \in \mathbb{R}$ as

$$
\widehat{\Lambda^{s} f}(\xi)=|\xi|^{s} \hat{f}(\xi) .
$$

For $s \in \mathbb{R}$, we define

$$
\|f\|_{\dot{H}^{s}}:=\left\|\Lambda^{s} f\right\|_{L^{2}}=\left(\int_{\mathbb{R}^{3}}|\xi|^{2 s}|\hat{f}(\xi)|^{2} \mathrm{~d} x\right)^{1 / 2}
$$

and the homogeneous Sobolev space $\dot{H}^{s}\left(\mathbb{R}^{3}\right):=\left\{f \in \mathcal{S}^{\prime}\left(\mathbb{R}^{3}\right):\|f\|_{\dot{H}^{s}}<\infty\right\}$. Similarly, the Sobolev space $H^{s, p}\left(\mathbb{R}^{3}\right)$ is equipped with the norm

$$
\|f\|_{\dot{H}^{s, p}}:=\left\|\Lambda^{s} f\right\|_{L^{p}} .
$$

Now we introduce the following bilinear commutator and the product estimates due to Kato-Ponce [12] and Kenig-Ponce-Vega [13]. 
Lemma 2.3. Let $s>0$ and $1<p<\infty$. If $f, g \in \dot{H}^{s, p}\left(\mathbb{R}^{3}\right)$, then there exists a constant $C$, independent of $f$ and $g$, such that

$$
\begin{aligned}
& \left\|\Lambda^{s}(f g)-f \Lambda^{s} g\right\|_{L^{p}} \leq C\left(\|\nabla f\|_{L^{p_{1}}}\left\|\Lambda^{s-1} g\right\|_{L^{q_{1}}}+\left\|\Lambda^{s} f\right\|_{L^{p_{2}}}\|g\|_{L^{q_{2}}}\right) \\
& \left\|\Lambda^{s}(f g)\right\|_{L^{p}} \leq C\left(\|f\|_{L^{p_{1}}}\left\|\Lambda^{s} g\right\|_{L^{q_{1}}}+\left\|\Lambda^{s} f\right\|_{L^{p_{2}}}\|g\|_{L^{q_{2}}}\right)
\end{aligned}
$$

where $p_{1}, p_{2} \in(1, \infty]$ satisfy

$$
\frac{1}{p}=\frac{1}{p_{1}}+\frac{1}{q_{1}}=\frac{1}{p_{2}}+\frac{1}{q_{2}}
$$

To end this section, we finally define the homogeneous Besov space $\dot{B}_{p, \infty}^{s}$.

Definition $2.4([26])$. Let $\varphi \in C^{\infty}\left(\mathbb{R}^{3}\right)$ be supported in some annulus $\mathcal{C} \subset$ $\mathbb{R}^{3}$ centered at 0 , say $\left\{\xi \in \mathbb{R}^{3}, 3 / 4 \leq|\xi| \leq 8 / 3\right\}$, such that $\sum_{q \in \mathbb{Z}} \varphi\left(2^{-q} \xi\right)=1$ for $\xi \neq 0$. Denoting $h=\mathcal{F}^{-1} \varphi$, we then define the dyadic blocks as

$$
\Delta_{q}:=\varphi\left(2^{-q} D\right) v=2^{3 q} \int_{\mathbb{R}^{3}} h\left(2^{q} y\right) v(x-y) \mathrm{d} y .
$$

For $s \in \mathbb{R}, p \in[1,+\infty]$, the homogeneous Besov space $\dot{B}_{p, \infty}^{s}$ is defined as

$$
\dot{B}_{p, \infty}^{s}:=\left\{v \in \mathcal{S}^{\prime}\left(\mathbb{R}^{3}\right),\|v\|_{B_{p, \infty}^{s}}:=\sup _{q \in \mathbb{Z}} 2^{s q}\left\|\Delta_{q} v\right\|_{L^{p}}<\infty \text { and } v=\sum_{q \in \mathbb{Z}} \Delta_{q} v\right\} .
$$

\section{Proof of Theorem 1.1}

As mentioned before, the local-in-time well-posedness has been established in [17]. Although the functional setting in [17] are somewhat different from the statements in Theorem 1.1, we can modify the arguments in [17] slightly to obtain our desired local results, and we omit the arguments here. Thus, to complete the proof of Theorem 1.1 , it is sufficient to prove the a priori estimates (1.8).

First, by the maximum principle, it follows from (1.1), (1.4), and (1.7) that

$$
m \leq \rho \leq M_{1}<\infty .
$$

Multiplying (1.2) by $u$, integrating the result over $\mathbb{R}^{3}$, and using (1.1) and (1.4), we have

$$
\frac{1}{2} \frac{\mathrm{d}}{\mathrm{d} t} \int \rho|u|^{2} \mathrm{~d} x+\int \mu|\nabla u|^{2} \mathrm{~d} x+\int(u \cdot \nabla) d \cdot \Delta d \mathrm{~d} x=0 .
$$

Multiplying (1.3) by $-\Delta d$, integrating the result over $\mathbb{R}^{3}$, and using the fact that $|d|=1$ implies $|\nabla d|^{2}=-d \Delta d$, we obtain that

$$
\begin{aligned}
& \frac{1}{2} \frac{\mathrm{d}}{\mathrm{d} t} \int|\nabla d|^{2} \mathrm{~d} x+\int|\Delta d|^{2} \mathrm{~d} x-\int(u \cdot \nabla) d \cdot \Delta d \mathrm{~d} x \\
= & -\int|\nabla d|^{2} d \cdot \Delta d \mathrm{~d} x=\int|d \cdot \Delta d|^{2} \mathrm{~d} x \leq \int|\Delta d|^{2} \mathrm{~d} x .
\end{aligned}
$$

Summing up (3.2) and (3.3) we infer that

$$
\frac{1}{2} \frac{\mathrm{d}}{\mathrm{d} t} \int\left\{\rho|u|^{2}+|\nabla d|^{2}\right\} \mathrm{d} x+\int \mu|\nabla u|^{2} \mathrm{~d} x \leq 0
$$


Applying the operator $\Lambda$ to (1.1), multiplying the result by $\Lambda \rho$, integrating over $\mathbb{R}^{3}$, and using (1.4) and (2.7), we infer that

$$
\begin{aligned}
\frac{1}{2} \frac{\mathrm{d}}{\mathrm{d} t} \int|\Lambda \rho|^{2} \mathrm{~d} x & =-\int[\Lambda(u \nabla \rho)-u \nabla \Lambda \rho] \Lambda \rho \mathrm{d} x \\
& \leq\|\Lambda(u \nabla \rho)-u \nabla \Lambda \rho\|_{L^{2}}\|\Lambda \rho\|_{L^{2}} \\
& \leq C\|\nabla u\|_{L^{\infty}}\|\nabla \rho\|_{L^{2}}^{2} \\
& \leq C\|u\|_{H^{s}}\|\nabla \rho\|_{L^{2}}^{2} \\
& \leq C\left\{\|u\|_{H^{s}}^{3}+\|\nabla \rho\|_{L^{2}}^{3}\right\} .
\end{aligned}
$$

Now, let

$$
\begin{aligned}
\theta & :=2 \max \left\{s-1, \frac{s-1}{s-\frac{5}{2}}, \frac{3}{2}\right\} \\
\Phi(t) & :=\int_{0}^{t}\left(1+\|\nabla \rho\|_{H^{s-1}}^{2}+\|u\|_{H^{s}}^{2}+\|\nabla d\|_{H^{s}}^{2}\right)^{\frac{3 \theta}{2}} \mathrm{~d} \tau .
\end{aligned}
$$

Then (3.5) gives

$$
\|\nabla \rho(t)\|_{L^{2}}^{2} \leq C\{1+\Phi(t)\} .
$$

Similarly, applying the operator $\Lambda^{s}$ to (1.1), multiplying the result by $\Lambda^{s} \rho$, integrating over $\mathbb{R}^{3}$, and using (1.4) and (2.7), we deduce that

$$
\begin{aligned}
\frac{1}{2} \frac{\mathrm{d}}{\mathrm{d} t} \int\left|\Lambda^{s} \rho\right|^{2} \mathrm{~d} x & =-\int\left[\Lambda^{s}(u \nabla \rho)-u \nabla \Lambda^{s} \rho\right] \Lambda^{s} \rho \mathrm{d} x \\
& \leq\left\|\Lambda^{s}(u \nabla \rho)-u \nabla \Lambda^{s} \rho\right\|_{L^{2}}\left\|\Lambda^{s} \rho\right\|_{L^{2}} \\
& \leq C\left(\|\nabla u\|_{L^{\infty}}\left\|\Lambda^{s} \rho\right\|_{L^{2}}+\|\nabla \rho\|_{L^{\infty}}\left\|\Lambda^{s} u\right\|_{L^{2}}\right)\left\|\Lambda^{s} \rho\right\|_{L^{2}} \\
& \leq C\left(\|\nabla u\|_{L^{\infty}}+\|\nabla \rho\|_{L^{\infty}}\right)\left(\left\|\Lambda^{s} \rho\right\|_{L^{2}}^{2}+\left\|\Lambda^{s} u\right\|_{L^{2}}^{2}\right) \\
& \leq C\left\{\|\nabla \rho\|_{H^{s-1}}^{3}+\|u\|_{H^{s}}^{3}\right\} .
\end{aligned}
$$

Integrating the above inequality over $\mathbb{R}^{3}$, and using the estimates (3.6) and (3.7) gives

$$
\|\nabla \rho(t)\|_{H^{s-1}}^{2} \leq C(1+\Phi(t))
$$

Multiplying (1.2) by $u_{t}$, integrating the result over $\mathbb{R}^{3}$, and using (1.4), (3.1), and (3.4), we get

$$
\begin{aligned}
& \frac{\mu}{2} \frac{\mathrm{d}}{\mathrm{d} t} \int|\nabla u|^{2} \mathrm{~d} x+\int \rho\left|u_{t}\right|^{2} \mathrm{~d} x \\
= & -\int(\nabla \cdot(\nabla d \odot \nabla d)+\rho u \cdot \nabla u) u_{t} \mathrm{~d} x \\
\leq & \left(\|\nabla d\|_{L^{2}}\|\Delta d\|_{L^{\infty}}+\|\rho\|_{L^{\infty}}\|u\|_{L^{2}}\|\nabla u\|_{L^{\infty}}\right)\left\|u_{t}\right\|_{L^{2}} \\
\leq & C\left\{\|\Delta d\|_{L^{\infty}}+\|\nabla u\|_{L^{\infty}}\right\}\left\|u_{t}\right\|_{L^{2}} \\
\leq & C\left\{\|u\|_{H^{s}}^{2}+\|\nabla d\|_{H^{s}}^{2}\right\}+\frac{1}{2} \int \rho\left|u_{t}\right|^{2} \mathrm{~d} x
\end{aligned}
$$

which yields

$$
\int_{0}^{t}\left\|u_{t}(\tau)\right\|_{L^{2}}^{2} \mathrm{~d} \tau \leq C(1+\Phi(t))
$$


Applying the operator $\Lambda^{s-1}$ to (1.2), multiplying the result by $\Lambda^{s-1} u_{t}$, integrating over $\mathbb{R}^{3}$, and using (1.4), we infer that

$$
\begin{aligned}
& \frac{\mu}{2} \frac{\mathrm{d}}{\mathrm{d} t} \int\left|\nabla \Lambda^{s-1} u\right|^{2} \mathrm{~d} x+\int \rho\left|\Lambda^{s-1} u_{t}\right|^{2} \mathrm{~d} x \\
= & -\int \Lambda^{s-1} \operatorname{div}(\nabla d \odot \nabla d) \cdot \Lambda^{s-1} u_{t} \mathrm{~d} x-\int \Lambda^{s-1}(\rho u \cdot \nabla u) \cdot \Lambda^{s-1} u_{t} \mathrm{~d} x \\
& -\int\left[\Lambda^{s-1}\left(\rho u_{t}\right)-\rho \Lambda^{s-1} u_{t}\right] \Lambda^{s-1} u_{t} \mathrm{~d} x \\
\leq & \left\{\left\|\Lambda^{s-1} \operatorname{div}(\nabla d \odot \nabla d)\right\|_{L^{2}}+\left\|\Lambda^{s-1}(\rho u \nabla u)\right\|_{L^{2}}\right. \\
\quad & \left.\quad+\left\|\Lambda^{s-1}\left(\rho u_{t}\right)-\rho \Lambda^{s-1} u_{t}\right\|_{L^{2}}\right\}\left\|\Lambda^{s-1} u_{t}\right\|_{L^{2}} \\
:= & \left\{I_{1}+I_{2}+I_{3}\right\}\left\|\Lambda^{s-1} u_{t}\right\|_{L^{2}} .
\end{aligned}
$$

By using (2.8), the term $I_{1}$ can be bounded by

$$
I_{1} \leq C\|\nabla d\|_{L^{\infty}}\|\nabla d\|_{H^{s}} \leq C\|\nabla d\|_{H^{s}}^{2} .
$$

Similarly, the term $I_{2}$ can be controlled by

$$
\begin{aligned}
I_{2} & \leq C\left\{\|\rho u\|_{L^{\infty}}\left\|\Lambda^{s} u\right\|_{L^{2}}+\left\|\Lambda^{s-1}(\rho u)\right\|_{L^{2}}\|\nabla u\|_{L^{\infty}}\right\} \\
& \leq C\left\{\|u\|_{H^{s}}^{2}+\left(\|\rho\|_{L^{\infty}}\left\|\Lambda^{s-1} u\right\|_{L^{2}}+\|u\|_{L^{\infty}}\left\|\Lambda^{s-1} \rho\right\|_{L^{2}}\right)\|\nabla u\|_{L^{\infty}}\right\} \\
& \leq C\left\{\|u\|_{H^{s}}^{2}+\|u\|_{H^{s}}^{2}\|\nabla \rho\|_{H^{s-1}}\right\} \\
& \leq C\left\{1+\|u\|_{H^{s}}^{3}+\|\nabla \rho\|_{H^{s-1}}^{3}\right\} .
\end{aligned}
$$

Using (2.7) and the Gagliardo-Nirenberg inequality (2.1), the term $I_{3}$ can be bounded as follows:

$$
\begin{aligned}
I_{3} & \leq C\|\nabla \rho\|_{L^{\infty}}\left\|\Lambda^{s-2} u_{t}\right\|_{L^{2}}+C\left\|\Lambda^{s-1} \rho\right\|_{L^{2}}\left\|u_{t}\right\|_{L^{\infty}} \\
& \leq C\|\nabla \rho\|_{H^{s-1}}\left(\left\|\Lambda^{s-2} u_{t}\right\|_{L^{2}}+\left\|u_{t}\right\|_{L^{\infty}}\right) \\
& \leq C\|\nabla \rho\|_{H^{s-1}}\left\{\left\|u_{t}\right\|_{L^{2}}^{\frac{1}{s-1}}\left\|\Lambda^{s-1} u_{t}\right\|_{L^{2}}^{\frac{s-2}{s-1}}+\left\|u_{t}\right\|_{L^{2}}^{\frac{s-5 / 2}{s-1}}\left\|\Lambda^{s-1} u_{t}\right\|_{L^{2}}^{\frac{3 / 2}{s-1}}\right\} \\
& \leq \frac{m}{8}\left\|\Lambda^{s-1} u_{t}\right\|_{L^{2}}+C\left\{\|\nabla \rho\|_{H^{s-1}}^{s-1}+\|\nabla \rho\|_{H^{s-1}}^{\frac{s-1}{s-5 / 2}}\right\}\left\|u_{t}\right\|_{L^{2}} .
\end{aligned}
$$

Recall that the constant $m$ is the lower bound of the density. Inserting the above estimates for $I_{1}, I_{2}$, and $I_{3}$ into (3.10), we obtain

$$
\begin{aligned}
& \frac{\mu}{2} \frac{\mathrm{d}}{\mathrm{d} t} \int\left|\nabla \Lambda^{s-1} u\right|^{2} d x+m \int\left|\Lambda^{s-1} u_{t}\right|^{2} \mathrm{~d} x \\
\leq & C+C\left\{\|\nabla d\|_{H^{s}}^{4}+\|u\|_{H^{s}}^{6}+\|\nabla \rho\|_{H^{s-1}}^{6}+\left(\|\nabla \rho\|_{H^{s-1}}^{2(s-1)}+\|\nabla \rho\|_{H^{s-1}}^{\frac{2(s-1)}{s-5 / 2}}\right)\left\|u_{t}\right\|_{L^{2}}^{2}\right\} .
\end{aligned}
$$

Integrating the above inequality over $[0, t]$ and using (3.8) and (3.9), we have

$$
\int_{0}^{t}\left\|\Lambda^{s-1} u_{t}(\tau)\right\|_{L^{2}}^{2} \mathrm{~d} \tau \leq C\left(1+\Phi(t)+\Phi^{2}(t)\right) .
$$

Applying the operator $\Lambda^{s}$ on (1.2), multiplying the result by $\Lambda^{s} u$, and integrating over $\mathbb{R}^{3}$, we derive that

$$
\frac{1}{2} \frac{\mathrm{d}}{\mathrm{d} t} \int \rho\left|\Lambda^{s} u\right|^{2} \mathrm{~d} x+\mu \int\left|\Lambda^{s+1} u\right|^{2} \mathrm{~d} x
$$




$$
\begin{aligned}
& =-\int \Lambda^{s} \operatorname{div}(\nabla d \odot \nabla d) \Lambda^{s} u \mathrm{~d} x-\int\left[\Lambda^{s}\left(\rho u_{t}\right)-\rho \Lambda^{s} u_{t}\right] \Lambda^{s} u \mathrm{~d} x \\
& \quad-\int\left[\Lambda^{s}(\rho u \nabla u)-\rho u \Lambda^{s} \nabla u\right] \Lambda^{s} u \mathrm{~d} x \\
& :=I_{4}+I_{5}+I_{6} .
\end{aligned}
$$

Using (2.8), the term $I_{4}$ can be bounded as

$$
\begin{aligned}
I_{4} & \leq C\|\nabla d\|_{L^{\infty}}\left\|\Lambda^{s+2} d\right\|_{L^{2}}\left\|\Lambda^{s} u\right\|_{L^{2}} \\
& \leq \frac{1}{8}\left\|\Lambda^{s+2} d\right\|_{L^{2}}^{2}+C\left\{\left\|\Lambda^{s} u\right\|_{L^{2}}^{4}+\|\nabla d\|_{H^{s}}^{4}\right\} .
\end{aligned}
$$

Similarly, the terms $I_{5}$ and $I_{6}$ can be bounded by

$$
\begin{aligned}
I_{5} & \leq C\left(\|\nabla \rho\|_{L^{\infty}}\left\|\Lambda^{s-1} u_{t}\right\|_{L^{2}}+\left\|\Lambda^{s} \rho\right\|_{L^{2}}\left\|u_{t}\right\|_{L^{\infty}}\right)\left\|\Lambda^{s} u\right\|_{L^{2}} \\
& \leq C\left\{\left\|u_{t}\right\|_{H^{s-1}}^{2}+\|\nabla \rho\|_{H^{s-1}}^{4}+\|u\|_{H^{s}}^{4}\right\}, \\
I_{6} & \leq C\left(\|\nabla(\rho u)\|_{L^{\infty}}\left\|\Lambda^{s} u\right\|_{L^{2}}+\left\|\Lambda^{s}(\rho u)\right\|_{L^{2}}\|\nabla u\|_{L^{\infty}}\right)\left\|\Lambda^{s} u\right\|_{L^{2}} \\
& \leq C\|\nabla \rho\|_{H^{s-1}}\|u\|_{H^{s}}^{3} \\
& \leq C\left\{\|\nabla \rho\|_{H^{s-1}}^{4}+\|u\|_{H^{s}}^{4}\right\} .
\end{aligned}
$$

Inserting the above estimates into (3.12), we have

$$
\begin{aligned}
& \frac{1}{2} \frac{\mathrm{d}}{\mathrm{d} t} \int \rho\left|\Lambda^{s} u\right|^{2} \mathrm{~d} x+\mu \int\left|\Lambda^{s+1} u\right|^{2} \mathrm{~d} x \\
\leq & \frac{1}{8}\left\|\Lambda^{s+2} d\right\|_{L^{2}}^{2}+C\left\{\|u\|_{H^{s}}^{4}+\|\nabla \rho\|_{H^{s-1}}^{4}+\|\nabla d\|_{H^{s}}^{4}+\left\|u_{t}\right\|_{H^{s-1}}^{2}\right\} .
\end{aligned}
$$

Applying the operator $\Lambda^{s}$ to (1.3), multiplying the result by $\Lambda^{s+2} d$, integrating over $\mathbb{R}^{3}$, and using (2.8), we have

$$
\begin{aligned}
& \frac{1}{2} \frac{\mathrm{d}}{\mathrm{d} t} \int\left|\Lambda^{s+1} d\right|^{2} \mathrm{~d} x+\int\left|\Lambda^{s+2} d\right|^{2} \mathrm{~d} x \\
= & -\int \Lambda^{s}(u \cdot \nabla d) \cdot \Lambda^{s+2} d \mathrm{~d} x+\int \Lambda^{s}\left(d|\nabla d|^{2}\right) \cdot \Lambda^{s+2} d \mathrm{~d} x \\
\leq & C\left\{\|\nabla d\|_{H^{s}}\|u\|_{H^{s}}\left\|\Lambda^{s+2} d\right\|_{L^{2}}+\|\nabla d\|_{H^{s}}^{3}\left\|\Lambda^{s+2} d\right\|_{L^{2}}\right\} \\
\leq & \frac{1}{8}\left\|\Lambda^{s+2} d\right\|_{L^{2}}^{2}+C\left\{\|\nabla d\|_{H^{s}}^{6}+\|u\|_{H^{s}}^{3}\right\} .
\end{aligned}
$$

Combining (3.13) with (3.14), integrating the result over $\mathbb{R}^{3}$, and using (3.9) and (3.11), we have

$$
\int\left|\Lambda^{s} u(t)\right|^{2}+\left|\Lambda^{s+1} d(t)\right|^{2} \mathrm{~d} x \leq C\left\{1+\Phi(t)+\Phi^{2}(t)\right\} .
$$

Due to (3.4), (3.7), (3.8), and (3.15), we conclude that

$$
\begin{aligned}
\frac{\mathrm{d}}{\mathrm{d} t}(1+\Phi(t)) & =\frac{\mathrm{d}}{\mathrm{d} t} \Phi(t)=\left(1+\|\nabla \rho\|_{H^{s-1}}^{2}+\|u\|_{H^{s}}^{2}+\|\nabla d\|_{H^{s}}^{2}\right)^{\frac{3 \theta}{2}} \\
& \leq C\left(1+\Phi(t)+\Phi^{2}(t)\right)^{\frac{3}{2} \theta} \\
& \leq C(1+\Phi(t))^{3 \theta},
\end{aligned}
$$

which yields (by the Osgood Lemma) that there exists a $T^{*}>0$ independent of $\mu$ such that $\Phi\left(T^{*}\right) \leq C$, and thus (1.8) holds. This completes the proof of Theorem 1.1. 


\section{Proof of Theorem 1.3}

Because the local existence of strong solutions can be obtained in a standard way, we only need to prove a priori estimates. By arguments similar to those in Theorem 1.1, we have

$$
0 \leq \rho \leq M_{2}<\infty
$$

and

$$
\sup _{0 \leq t \leq T} \int\left\{\rho|u|^{2}+|\nabla d|^{2}\right\} \mathrm{d} x+\int_{0}^{T} \int \mu|\nabla u|^{2} \mathrm{~d} x \mathrm{~d} t \leq C .
$$

Multiplying (1.2) by $\partial_{t} u$, integrating the result over $\mathbb{R}^{3}$, using (1.1) and (1.4), we derive that

$$
\begin{aligned}
& \frac{\mu}{2} \frac{\mathrm{d}}{\mathrm{d} t} \int|\nabla u|^{2} \mathrm{~d} x+\int \rho\left|\partial_{t} u\right|^{2} \mathrm{~d} x \\
= & -\int \rho u \cdot \nabla u \cdot \partial_{t} u \mathrm{~d} x+\int \nabla \partial_{t} u:(\nabla d \odot \nabla d) \mathrm{d} x \\
:= & I I_{1}+I I_{2} .
\end{aligned}
$$

Using (4.1), Hölder's inequality, Young's inequality, and the Gagliardo-Nirenberg inequality (2.3), the term $I I_{1}$ can be bounded as

$$
\begin{aligned}
I I_{1} & \leq C\left\|\sqrt{\rho} \partial_{t} u\right\|_{L^{2}}\|u\|_{L^{s}}\|\nabla u\|_{L^{\frac{2 s}{s-2}}} \\
& \leq \epsilon\left\|\sqrt{\rho} \partial_{t} u\right\|_{L^{2}}^{2}+C\|u\|_{L^{s}}^{2}\|\nabla u\|_{L^{\frac{2 s}{s-2}}}^{2} \\
& \leq \epsilon\left\{\left\|\sqrt{\rho} \partial_{t} u\right\|_{L^{2}}^{2}+\|\Delta u\|_{L^{2}}^{2}\right\}+C\|u\|_{L^{s}}^{\frac{2 s}{s-3}}\|\nabla u\|_{L^{2}}^{2}
\end{aligned}
$$

where $0<\epsilon<1$ is a sufficient small constant.

On the other hand, because $(u, \pi)$ is a solution of the Stokes system,

$$
-\mu \Delta u+\nabla \pi=f:=-\nabla \cdot(\nabla d \odot \nabla d)-\rho \partial_{t} u-\rho u \cdot \nabla u .
$$

It follows from the $H^{2}$-theory of the Stokes system (for example, see [27]) that

$$
\begin{aligned}
\left\|\nabla^{2} u\right\|_{L^{2}} & \leq C\|f\|_{L^{2}} \\
\leq C\left\{\|\nabla d\|_{L^{4}}\|\Delta d\|_{L^{4}}+\left\|\sqrt{\rho} \partial_{t} u\right\|_{L^{2}}+\|u\|_{L^{s}}\|\nabla u\|_{L^{\frac{2 s}{s-2}}}\right\} & \leq C\|\Delta d\|_{L^{2}}^{1 / 2}\|\nabla d\|_{\dot{B}_{\infty, \infty}^{0}}^{1 / 2}\|\nabla \Delta d\|_{L^{2}}^{1 / 2}+C\left\|\sqrt{\rho} \partial_{t} u\right\|_{L^{2}} \\
& \quad+C\|u\|_{L^{s}}\|\nabla u\|_{L^{2}}^{1-3 / s}\|\Delta u\|_{L^{2}}^{3 / s},
\end{aligned}
$$

where (4.1), Hölder's inequality, the Gagliardo-Nirenberg inequality (2.2), and the elegant Machihara-Ozawa inequality [23] (also see Meyer [24])

$$
\|\nabla u\|_{L^{4}}^{2} \leq C\|u\|_{\dot{B}_{\infty, \infty}^{0}}\|\Delta u\|_{L^{2}}
$$

are used. Hence

$$
\left\|\nabla^{2} u\right\|_{L^{2}} \leq C\|\Delta d\|_{L^{2}}^{1 / 2}\|\nabla d\|_{\dot{B}_{\infty, \infty}^{0}}^{1 / 2}\|\nabla \Delta d\|_{L^{2}}^{1 / 2}
$$




$$
+C\left\|\sqrt{\rho} \partial_{t} u\right\|_{L^{2}}+C\|u\|_{L^{s}}^{\frac{s}{s-3}}\|\nabla u\|_{L^{2}} .
$$

By integration by parts, the term $I I_{2}$ can be rewritten as

$$
\begin{aligned}
I_{2} & =\frac{\mathrm{d}}{\mathrm{d} t} \int \nabla u: \nabla d \odot \nabla d \mathrm{~d} x-2 \int \nabla u: \nabla d \odot \partial_{t} \nabla d \mathrm{~d} x \\
& =\frac{\mathrm{d}}{\mathrm{d} t} \int \nabla u: \nabla d \odot \nabla d \mathrm{~d} x-2 \sum_{i, j=1}^{3} \int \partial_{i} u_{j} \partial_{i} d \partial_{j} \partial_{t} d \mathrm{~d} x \\
& =\frac{\mathrm{d}}{\mathrm{d} t} \int \nabla u: \nabla d \odot \nabla d \mathrm{~d} x+2 \sum_{i, j=1}^{3} \int \partial_{i} u_{j} \partial_{i} \partial_{j} d \partial_{t} d \mathrm{~d} x \\
& :=\frac{\mathrm{d}}{\mathrm{d} t} \int \nabla u: \nabla d \odot \nabla d \mathrm{~d} x+2 I I_{3} .
\end{aligned}
$$

Applying the equation (1.3), Hölder's inequality, the Gagliardo-Nirenberg inequalities (2.2) and (2.3), and (4.5), we obtain that

$$
\begin{aligned}
I I_{3}= & \sum_{i, j=1}^{3} \int \partial_{i} u_{j} \partial_{i} \partial_{j} d\left(\Delta d+d|\nabla d|^{2}-u \cdot \nabla d\right) \mathrm{d} x \\
\leq & C\left\{\|\nabla u\|_{L^{2}}\left(\|\Delta d\|_{L^{4}}^{2}+\|\nabla d\|_{L^{8}}^{2}\right)+\|\nabla u\|_{L^{\frac{2 s}{s-2}}}\|u\|_{L^{s}}\|\Delta d\|_{L^{4}}\|\nabla d\|_{L^{4}}\right\} \\
\leq & C\left\{\|\nabla u\|_{L^{2}}\|\Delta d\|_{L^{4}}^{2}+C\|\nabla u\|_{L^{\frac{2 s}{s-2}}}^{2}\|u\|_{L^{s}}^{2}+C\|\Delta d\|_{L^{2}}\|\nabla d\|_{\dot{B}_{\infty, \infty}^{0}}\|\nabla \Delta d\|_{L^{2}}\right\} \\
\leq & C\left\{\|\nabla u\|_{L^{2}}\|\nabla d\|_{\dot{B}_{\infty, \infty}^{0}}\|\nabla \Delta d\|_{L^{2}}+\|u\|_{L^{s}}^{2}\|\nabla u\|_{L^{2}}^{2\left(1-\frac{3}{s}\right)}\|\Delta u\|_{L^{2}}^{3 / s}\right. \\
& \left.\quad+\|\Delta d\|_{L^{2}}\|\nabla d\|_{\dot{B}_{\infty, \infty}^{0}}\|\nabla \Delta d\|_{L^{2}}\right\} \\
\leq & \epsilon\left\{\|\nabla \Delta d\|_{L^{2}}^{2}+\|\Delta u\|_{L^{2}}^{2}\right\}+C\|\nabla d\|_{\dot{B}_{\infty, \infty}^{0}}^{2}\left(\|\nabla u\|_{L^{2}}^{2}+\|\Delta d\|_{L^{2}}^{2}\right) \\
& +C\|u\|_{L^{s}}^{\frac{2 s}{s-3}}\|\nabla u\|_{L^{2}}^{2},
\end{aligned}
$$

where $0<\epsilon<1$ is a sufficient small constant.

Applying the operator $\Delta$ to (1.3), multiplying the result by $\Delta d$, and integrating over $\mathbb{R}^{3}$, we get

$$
\begin{aligned}
& \frac{1}{2} \frac{\mathrm{d}}{\mathrm{d} t} \int|\Delta d|^{2} \mathrm{~d} x+\int|\nabla \Delta d|^{2} \mathrm{~d} x \\
\leq & \left|\int \Delta(u \cdot \nabla d) \cdot \Delta d \mathrm{~d} x\right|+\sum_{k=1}^{3}\left|\int \partial_{k}\left(d|\nabla d|^{2}\right) \partial_{k} \Delta d \mathrm{~d} x\right| \\
: & =J_{1}+J_{2} .
\end{aligned}
$$

Using Hölder's inequality, Young's inequality, the Gagliardo-Nirenberg inequalities (2.2) and (2.3), and (4.5), the terms $J_{1}$ and $J_{2}$ can be estimated as follows:

$$
\begin{aligned}
J_{1} & \leq\left|\int \Delta u \cdot \nabla d \cdot \Delta d \mathrm{~d} x\right|+2\left|\sum_{k=1}^{3} \int \partial_{k} u \cdot \partial_{k} \nabla d \cdot \Delta d \mathrm{~d} x\right| \\
& \leq\|\Delta u\|_{L^{2}}\|\nabla d\|_{L^{4}}\|\Delta d\|_{L^{4}}+C\|\nabla u\|_{L^{2}}\|\Delta d\|_{L^{4}}^{2} \\
& \leq \epsilon\|\Delta u\|_{L^{2}}^{2}+C\left\{\|\Delta d\|_{L^{2}}\|\nabla d\|_{\dot{B}_{\infty, \infty}^{0}}\|\nabla \Delta d\|_{L^{2}}\right.
\end{aligned}
$$




$$
\begin{gathered}
\left.\quad+\|\nabla u\|_{L^{2}}\|\nabla d\|_{\dot{B}_{\infty, \infty}^{0}}\|\nabla \Delta d\|_{L^{2}}\right\} \\
\leq \epsilon\|\Delta u\|_{L^{2}}^{2}+\epsilon\|\nabla \Delta d\|_{L^{2}}^{2}+C\|\nabla d\|_{\dot{B}_{\infty, \infty}^{0}}^{2}\left(\|\nabla u\|_{L^{2}}^{2}+\|\Delta d\|_{L^{2}}^{2}\right), \\
J_{2} \leq\left.\sum_{k=1}^{3}\left|\int \partial_{k} d\right| \nabla d\right|^{2} \partial_{k} \Delta d \mathrm{~d} x\left|+\sum_{k=1}^{3}\right| \int d \partial_{k}|\nabla d|^{2} \partial_{k} \Delta d \mathrm{~d} x \mid \\
\leq \sum_{k=1}^{3}\left|\int \partial_{k}\left(\partial_{k} d|\nabla d|^{2}\right) \cdot \Delta d \mathrm{~d} x\right|+\left.\sum_{k=1}^{3}\left|\int d \partial_{k}\right| \nabla d\right|^{2} \partial_{k} \Delta d \mathrm{~d} x \mid \\
\leq C\left\{\int|\nabla d|^{2}\left|\nabla^{2} d\right|^{2} \mathrm{~d} x+\int|\nabla d| \cdot|\Delta d| \cdot|\nabla \Delta d| \mathrm{d} x\right\} \\
\leq C\left\{\|\nabla d\|_{L^{4}}^{2}\|\Delta d\|_{L^{4}}^{2}+\|\nabla d\|_{L^{4}}\|\Delta d\|_{L^{4}}\|\nabla \Delta d\|_{L^{2}}\right\} \\
\leq \epsilon\|\nabla \Delta d\|_{L^{2}}^{2}+C\|\Delta d\|_{L^{2}}\|\nabla d\|_{\dot{B}_{\infty, \infty}^{0}}\|\nabla \Delta d\|_{L^{2}} \\
\leq 2 \epsilon\|\nabla \Delta d\|_{L^{2}}^{2}+C\|\nabla d\|_{\dot{B}_{\infty, \infty}^{0}}^{2}\|\Delta d\|_{L^{2}}^{2}
\end{gathered}
$$

where $0<\epsilon<1$ is a sufficient small constant.

Combining (4.3), (4.6), (4.7), (4.8), (4.9), (4.10), and (4.11), taking $\epsilon$ small enough, noting that

$$
\left|\int \nabla u: \nabla d \otimes \nabla d \mathrm{~d} x\right| \leq\|\nabla u\|_{L^{2}}\|\nabla d\|_{L^{4}}^{2} \leq C\|\nabla u\|_{L^{2}}\|\Delta d\|_{L^{2}},
$$

and using the Gronwall inequality, we get that

$$
\begin{aligned}
& \|\nabla u\|_{L^{\infty}\left(0, T ; L^{2}\right)} \leq C, \quad\|\nabla u\|_{L^{2}\left(0, T ; H^{1}\right)} \leq C, \quad\left\|\sqrt{\rho} \partial_{t} u\right\|_{L^{2}\left(0, T ; L^{2}\right)} \leq C \\
& \|\nabla d\|_{L^{\infty}\left(0, T ; H^{1}\right)}+\|\nabla d\|_{L^{2}\left(0, T ; H^{2}\right)} \leq C .
\end{aligned}
$$

It follows from (1.3), (4.12), and (4.13) that

$$
\left\|\partial_{t} d\right\|_{L^{\infty}\left(0, T ; L^{2}\right)} \leq C, \quad\left\|\partial_{t} d\right\|_{L^{2}\left(0, T ; H^{1}\right)} \leq C .
$$

Applying the operator $\partial_{t}$ to (1.2), integrating the result over $\mathbb{R}^{3}$, and using (1.1), (1.4), (4.12), and (4.13), we obtain that

$$
\begin{aligned}
& \quad \frac{1}{2} \frac{\mathrm{d}}{\mathrm{d} t} \int \rho\left|\partial_{t} u\right|^{2} \mathrm{~d} x+\mu \int\left|\nabla \partial_{t} u\right|^{2} \mathrm{~d} x \\
& \leq C \int|\nabla d|\left|\partial_{t} \nabla d\right|\left|\nabla \partial_{t} u\right| \mathrm{d} x+\left|\int \rho u \cdot \nabla\left[\left(\partial_{t} u+u \cdot \nabla u\right) \partial_{t} u\right] \mathrm{d} x\right| \\
& +C\left|\int \rho \partial_{t} u \cdot \nabla u \cdot \partial_{t} u \mathrm{~d} x\right| \\
& \leq C\left\{\|\nabla d\|_{L^{\infty}}\left\|\partial_{t} \nabla d\right\|_{L^{2}}\left\|\nabla \partial_{t} u\right\|_{L^{2}}+\left\|\sqrt{\rho} \partial_{t} u\right\|_{L^{2}}\|u\|_{L^{\infty}}\left\|\nabla \partial_{t} u\right\|_{L^{2}}\right. \\
& \quad+\|u\|_{L^{6}}^{2}\|\nabla u\|_{L^{6}}\left\|\nabla \partial_{t} u\right\|_{L^{2}}+\left\|\sqrt{\rho} \partial_{t} u\right\|_{L^{2}}\|u\|_{L^{6}}\|\nabla u\|_{L^{6}}^{2} \\
& \left.\quad+\|u\|_{L^{6}}^{2}\|\Delta u\|_{L^{2}}\left\|\partial_{t} u\right\|_{L^{6}}+\left\|\sqrt{\rho} \partial_{t} u\right\|_{L^{2}}\|\nabla u\|_{L^{3}}\left\|\partial_{t} u\right\|_{L^{6}}\right\} \\
& \leq \frac{1}{8}\left\|\nabla \partial_{t} u\right\|_{L^{2}}^{2}+C\left\{\|\nabla d\|_{L^{\infty}}\left\|\partial_{t} \nabla d\right\|_{L^{2}}^{2}+\|u\|_{L^{\infty}}^{2}\left\|\sqrt{\rho} \partial_{t} u\right\|_{L^{2}}^{2}\right. \\
& \left.\quad+\|\Delta u\|_{L^{2}}^{2}+\|\Delta u\|_{L^{2}}^{2}\left\|\sqrt{\rho} \partial_{t} u\right\|_{L^{2}}+\|\nabla u\|_{L^{3}}^{2}\left\|\sqrt{\rho} \partial_{t} u\right\|_{L^{2}}^{2}\right\} .
\end{aligned}
$$


Applying the operator $\partial_{t}$ to (1.3), multiplying the result by $\partial_{t} \Delta d$, integrating over $\mathbb{R}^{3}$, and using (4.12) and (4.13), we have

$$
\begin{aligned}
& \frac{1}{2} \frac{\mathrm{d}}{\mathrm{d} t} \int\left|\nabla \partial_{t} d\right|^{2} \mathrm{~d} x+\int\left|\Delta \partial_{t} d\right|^{2} \mathrm{~d} x \\
\leq & \left|\int\left(\partial_{t} u \cdot \nabla d+u \cdot \nabla \partial_{t} d\right) \Delta \partial_{t} d \mathrm{~d} x\right|+\int\left(\partial_{t} d|\nabla d|^{2}+d \partial_{t}|\nabla d|^{2}\right) \Delta \partial_{t} d \mathrm{~d} x \\
\leq & C\left\{\left(\left\|\partial_{t} u\right\|_{L^{6}}\|\nabla d\|_{L^{3}}+\|u\|_{L^{6}}\left\|\nabla \partial_{t} d\right\|_{L^{3}}\right)\left\|\Delta \partial_{t} d\right\|_{L^{2}}\right. \\
\quad & \left.\quad+\left(\left\|\partial_{t} d\right\|_{L^{6}}\|\nabla d\|_{L^{6}}+\|d\|_{L^{\infty}}\|\nabla d\|_{L^{6}}\left\|\partial_{t} \nabla d\right\|_{L^{3}}\right)\left\|\Delta \partial_{t} d\right\|_{L^{2}}\right\} \\
\leq & C\left(\left\|\nabla \partial_{t} u\right\|_{L^{2}}+\left\|\nabla \partial_{t} d\right\|_{L^{2}}+\left\|\nabla \partial_{t} d\right\|_{L^{3}}\right)\left\|\Delta \partial_{t} d\right\|_{L^{2}} \\
\leq & \frac{1}{8}\left\|\Delta \partial_{t} d\right\|_{L^{2}}^{2}+C\left\{\left\|\nabla \partial_{t} u\right\|_{L^{2}}^{2}++\left\|\nabla \partial_{t} d\right\|_{L^{2}}^{2}\right\}
\end{aligned}
$$

where the Hölder inequality and the Gagliardo-Nirenberg inequalities (2.4) and (2.5) are used.

Combining (4.15) with (4.16), and using the Gronwall inequality, we have

$$
\begin{aligned}
& \left\|\sqrt{\rho} \partial_{t} u\right\|_{L^{\infty}\left(0, T ; L^{2}\right)}+\left\|\nabla \partial_{t} u\right\|_{L^{2}\left(0, T ; L^{2}\right)} \leq C, \\
& \left\|\partial_{t} d\right\|_{L^{\infty}\left(0, T ; H^{1}\right)}+\left\|\partial_{t} d\right\|_{L^{2}\left(0, T ; H^{2}\right)} \leq C .
\end{aligned}
$$

It follows from (1.3), (4.12), (4.13), (4.18), and the Gagliardo-Nirenberg inequality (2.6) that

$$
\begin{aligned}
\|\nabla \Delta d\|_{L^{2}} \leq C\left\|\nabla \partial_{t} d+\nabla u \cdot \nabla d+u \cdot \nabla^{2} d-\nabla\left(d|\nabla d|^{2}\right)\right\|_{L^{2}} \\
\leq C+C\left\{\|\nabla u\|_{L^{2}}\|\nabla d\|_{L^{\infty}}+\|u\|_{L^{6}}\|\Delta d\|_{L^{3}}\right. \\
\left.\quad \quad+\|\nabla d\|_{L^{\infty}}\|\Delta d\|_{L^{2}}+\|\nabla d\|_{L^{6}}^{3}\right\} \\
\leq C+C\left\{\|\nabla d\|_{L^{\infty}}+\|\Delta d\|_{L^{3}}\right\} \\
\leq C+C\left\{\|\nabla d\|_{L^{6}}^{1 / 2}\|\nabla \Delta d\|_{L^{2}}^{1 / 2}+\|\Delta d\|_{L^{2}}^{1 / 2}\|\nabla \Delta d\|_{L^{2}}^{1 / 2}\right\},
\end{aligned}
$$

which gives

$$
\|\nabla d\|_{L^{\infty}\left(0, T ; H^{2}\right)} \leq C .
$$

Similarly, we can prove that

$$
\|\nabla u\|_{L^{\infty}\left(0, T ; H^{1}\right)}+\|\nabla u\|_{L^{2}\left(0, T ; W^{1,6}\right)} \leq C .
$$

Applying the operator $\partial_{i}$ to $(1.1)$, multiplying the result by $\left|\partial_{i} \rho\right|^{q-2} \partial_{i} \rho$, integrating over $\mathbb{R}^{3}$, summing over $i$, and using (1.4) and (4.20), we conclude that

$$
\frac{\mathrm{d}}{\mathrm{d} t} \int|\nabla \rho|^{q} \mathrm{~d} x \leq C\|\nabla u\|_{L^{\infty}} \int|\nabla \rho|^{q} \mathrm{~d} x \leq C\|\nabla u\|_{W^{1,6}}\|\nabla \rho\|_{L^{q}}^{q},
$$

which implies

$$
\|\nabla \rho\|_{L^{\infty}\left(0, T ; L^{2} \cap L^{q}\right)} \leq C .
$$

This completes the proof of Theorem 1.3.

Acknowledgment. The authors are very grateful to the referees for their constructive comments which improved the earlier version of this paper. Fan was supported by NSFC (No. 11171154). Li was supported by NSFC (Grant No. 11271184), NCET-11-0227, PAPD, and the Fundamental Research Funds for the Central Universities. 


\section{REFERENCES}

[1] S. Chandrasekhar, Liquid Crystals, Second Edition, Cambridge Unversity Press, 1992.

[2] R. Danchin, The inviscid limit for density-dependent incompressible fluids, Ann. Fac. Sci. Toulouse Math., (6), 15(4), 637-688, 2006.

[3] R. Danchin, Density-dependent incompressible viscous fluids in critical spaces, Proc. Roy. Soc. Edinburgh Sect. A, 133(6), 1311-1334, 2003.

[4] R. Danchin and F. Fanelli, The well-posedness issue for the density-dependent Euler equations in endpoint Besov spaces, J. Math. Pures Appl., (9), 96(3), 253-278, 2011.

[5] R. Danchin and P.B. Mucha, A Lagrangian approach for the incompressible Navier-Stokes equations with variable density, Commun. Pure Appl. Math., 65(10), 1458-1480, 2012.

[6] J.L. Ericksen, Hydrostatic theory of liquid crystal, Arch. Rat. Mech. Anal., 9, 371-378, 1962.

[7] J. Fan, H. Gao, and B. Guo, Regularity criteria for the Navier-Stokes-Landau-Lifshitz system, J. Math. Anal. Appl., 363(1), 29-37, 2010.

[8] T.M. Fleet, Differential Analysis, Cambridge University Press, 1980.

[9] E. Gagliardo, Proprietà di alcune classi di funzioni in più variabili, Ricerche di. Matem., 7, 102-137, 1958.

[10] T. Huang, C.Y. Wang, and H.Y. Wen, Blow up criterion for compressible nematic liquid crystal flows in dimension three, Arch. Rat. Mech. Anal., 204, 285-311, 2012.

[11] S. Itoh, On the vanishing viscosity in the Cauchy problem for the equations of a nonhomogeneous incompressible fluid, Glasgow Math. J., 36, 123-129, 1994.

[12] T. Kato and G. Ponce, Commutator estimates and the Euler and Navier-Stokes equations, Commun. Pure Appl. Math., 41, 891-907, 1988.

[13] C. Kenig, G. Ponce, and L. Vega, Well-posedness of the initial value problem for the Kortewegde Vries equation, J. Amer. Math. Soc., 4, 323-347, 1991.

[14] H. Kim, A blow-up criterion for the nonhomogeneous incompressible Navier-Stokes equations, SIAM J. Math. Anal., 37, 1417-1434, 2006.

[15] F.M. Leslie, Some constitute equations for liquid crystals, Arch. Rat. Mech. Anal., 28, 265-283, 1968.

[16] X. Li and D. Wang, Global solution to the incompressible flow of liquid crystals, J. Diff. Eqs., $252,745-767,2012$.

[17] X. Li and D. Wang, Global strong solution to the density-dependent incompressible flow of liquid crystals, Trans. Amer. Math. Soc., accepted. Available at arXiv: 1202.1011v1 [math.AP].

[18] F.-H. Lin, Nonlinear theory of defects in nematic liquid crystals: Phase transition and flow phenomena, Commun. Pure. Appl. Math., 42, 789-814, 1989.

[19] F.-H. Lin and C. Liu, Nonparabolic dissipative systems modeling the flow of liquid crystals, Commun. Pure Appl. Math., 48, 501-537, 1995.

[20] F.-H. Lin and C. Liu, Static and dynamic theories of liquid crystals, J. Part. Diff. Eqs., 14, 289-330, 2001.

[21] F.-H. Lin and C. Liu, Partial regularity of the dynamic system modeling the flow of liquid crystals, Disc. Cont. Dyn. Syst., 2, 1-22, 1996.

[22] F.-H. Lin and C. Liu, Existence of solutions for the Ericksen-Leslie system, Arch. Rat. Mech. Anal., 154, 135-156, 2000.

[23] S. Machihara and T. Ozawa, Interpolation inequalities in Besov spaces, Proc. Amer. Math. Soc., 131(5), 1553-1556, 2002.

[24] Y. Meyer, Oscillating patterns in some nonlinear evolution equations, in Mathematical Foundation of Turbulent Viscous Flows, M. Cannone and T. Miyakawa (eds.), Lecture Notes in Math., Springer-Verlag, Berlin, 1871, 101-187, 2006.

[25] L. Nirenberg, On elliptic partial differential equations, Ann. Scuola Norm. Sup. Pisa (3), 13, 115-162, 1959.

[26] J. Peetre, New Thoughts on Besov Spaces, Duke University Mathematical Series, Durham N. C, 1, 1976.

[27] R. Temam, Navier-Stokes Equations, Theory and numerical analysis, Third Edition, North Holland Publishing Co., Amsterdam-New York, 1984 